\title{
Fibre Orientation Modelling for Rubber Press Forming of Thermoplastic Laminates
}

\author{
E.A.D. Lamers, R.Akkerman* \& S. Wijskamp \\ University of Twente, Department of Mechanical Engineering, Composites Group, PO box 217, 7500 AE \\ Enschede, the Netherlands \\ URL: www.composites.wb.utwente.nl \\ e-mail:E.A.D.Lamers@ctw.utwente.nl;.R.Akkerman@ctw.utwente.nl;.S.Wijskamp@ctw.utwente.nl
}

In order to predict product distortions when producing thermoplastic composite parts in the Rubber Press Forming process, the occurring fibre re-orientation must be known. Classical geometric drape simulation methods can be used to predict the fibre re-orientation. However, these methods have difficulties to incorporate the process specific boundary conditions. Therefore, a fabric reinforced fluid model was implemented in the Finite Element package DIEKA, which is capable of modelling the boundary conditions as well. The predictions of both types of simulations are compared with the results of drape experiments on a double dome geometry.

Key words: composites, draping, FE-draping, material forming

\section{INTRODUCTION}

Thermoplastic composites have a good potential to improve the cost-effectiveness of advanced polymer composites. Hot pressing of these materials offers a significantly higher production rate than the autoclave processes, increasing the economical competitiveness to other construction materials such as for example aluminium.

A relatively fast process is the Rubber Press Forming process (RPF) for fabric-reinforced thermoplastics. Typically, the production cycle is in the order of a few minutes and consists of infrared heating of a multi-layer preform above melt or glass transition temperature, hot pressing of the preform and cooling the preform below the glass transition temperature of the thermoplastic matrix.

In the process, the negatively shaped rubber upper mould presses the hot preform on a positively shaped steel lower mould. During pressing the rubber deforms nearly hydrostatically, proving a good way to consolidate the laminate.

Heating, pressing, cooling and removing the product from the press are automated, ensuring reproducibility. Due to the good draping characteristics of the fabric material, the processing forces are low and the tools are relatively inexpensive.
However, during production shrinking and warpage may result in unacceptable dimensional changes of the products. Fibre re-orientation is one of the major factors causing these distortions. Especially when producing doubly curved parts, the process of draping causes the angle between the warp and weft yarns to vary over the product. As a result of this reorientation, the thermomechanical properties of the fibre reinforced composite material show a corresponding distribution, in turn leading to a distribution of residual stresses. Obviously, the resulting fibre orientations must be predicted accurately to predict the overall thermomechanical properties and distortions of the product.

In the past, draping has been modelled using several methods. A widely used method is geometrical draping. Geometrical draping methods however are nearly incapable of modelling process specific boundary conditions. To incorporate these conditions FE simulations are used to predict the fibre re-orientation.

Here, a fabric reinforced fluid was implemented in DIEKA, a Finite Element (FE) package used for modelling forming processes, such as e.g. deep drawing of metals [1]. Using different lay-ups, drape experiments were performed on a double dome geometry. Finally, the FE-model and the geometrical draping method are compared with experimental results. 


\section{GEOMETRICAL DRAPING}

The geometrical draping approach is commonly used to predict the resulting fibre re-orientation for doubly curved fabric reinforced products. Typically, draping starts from an initial point and two initial fibre directions. Further points are then generated at a fixed equal distance from the previous points. By applying certain "strategies" (Bergsma [2]), different drape solutions can be found. The method does not include any restraining conditions from the tools and is nearly incapable of incorporating the fabric properties. Especially in tight weaves, the error of assuming a zero in-plane fabric shear stiffness during draping leads to errors in predicting the resulting fibre distribution. The resin material also affects the deformation properties. In addition, the geometrical draping approach might find infeasible solutions when draping products with holes.

\section{FINITE ELEMENT DRAPING}

Several authors $[\mathbf{3}, \mathbf{4}]$ modelled the draping process using FE methods. One of the main reasons for this was the incapability of the geometrical method to incorporate the composite properties and processing conditions during draping.

\subsection{Constitutive model}

In DIEKA, a material model similar to the model developed by Spencer [5] was implemented. In his work, Spencer assumes the fibres to be inextensible, while the matrix material is incompressible. These rather strong conditions were adapted for the FE simulation by assuming finite fibre stiffness in the fibre direction, and compressible matrix properties. The fibres are assumed to have no stiffness properties in other directions while the matrix response is Newtonian viscous. The stress is determined as:

$\boldsymbol{\sigma}=-p \boldsymbol{I}+S_{a} V_{f 1} \boldsymbol{A}+S_{b} V_{f 2} \boldsymbol{B}+V_{m} \boldsymbol{\tau}(\boldsymbol{D}, \boldsymbol{a}, \boldsymbol{b})$

where

$$
\begin{aligned}
\boldsymbol{\tau}(\boldsymbol{D}, \boldsymbol{a}, \boldsymbol{b}) & =2 \eta \boldsymbol{D}+2 \eta_{1}(\boldsymbol{A} \cdot \boldsymbol{D}+\boldsymbol{D} \cdot \boldsymbol{A})+ \\
& 2 \eta_{2}(\boldsymbol{B} \cdot \boldsymbol{D}+\boldsymbol{D} \cdot \boldsymbol{B})+2 \eta_{3}\left(\boldsymbol{C} \cdot \boldsymbol{D}+\boldsymbol{D} \cdot \boldsymbol{C}^{T}\right)+ \\
& 2 \eta_{4}\left(\boldsymbol{C}^{T} \cdot \boldsymbol{D}+\boldsymbol{D} \cdot \boldsymbol{C}\right)
\end{aligned}
$$

and

$$
\boldsymbol{A}=\boldsymbol{a} \boldsymbol{a}, \boldsymbol{B}=\boldsymbol{b} \boldsymbol{b}, \boldsymbol{C}=\boldsymbol{a b}
$$

In these equations, $\sigma$ and $\tau$ are stresses, $\boldsymbol{D}$ is the rate of deformation tensor, $S_{a, b}$ are the fibre longitudinal stresses (which depend on the fibre longitudinal strains and moduli only), while $\boldsymbol{a}$ and $\boldsymbol{b}$ are unit vectors in the fibre directions. $\eta, \eta_{1}, \eta_{2}, \eta_{3}$ and $\eta_{4}$ are matrix viscosities that depend on the angle between the fibre directions. $V_{f 1}, V_{f 2}$ and $V_{m}$ are the volume fractions of the constituents.

\subsection{Implementation of the FE-model}

Linear triangular membrane elements with one integration point were used to implement the material model in DIEKA. When assuming plane stress, the matrix material effectively becomes incompressible without the capability of introducing deviatoric stresses.

Contact between both sides of the composite and the moulds was modelled using six node wedge contact elements. On the steel face, the normal contact stiffness was high, while on the rubber face, the normal contact stiffness was relatively low. Viscous sliding friction was used with a constant friction coefficient. The traction due to friction is defined as $\boldsymbol{t}=f \boldsymbol{V}_{\text {rel }}$

where $f$ is the constant friction coefficient and $\boldsymbol{V}_{\text {rel }}$ is the difference in velocity between the contact surfaces.

Representing the fibre directions in natural coordinates proved a convenient way to describe the fibre distortions in the updated Lagrange discretisation. In natural coordinates, the fibre orientations remain constant, so in global coordinates these automatically conform to the elements distortions.

The drape simulation is displacement controlled by moving the steel mould towards the rubber mould in small steps. For each displacement step, the system is solved using a predictor-corrector scheme (implicit). The simulation stops when the steel mould reaches the bottom of the rubber mould. In reality, the pressing cycle does not stop at this point. The closed moulds are then firmly pressed together under load control to consolidate the laminate. However, it is assumed that this part of the pressing cycle does not influence the fibre re-orientation and therefore this final step is not simulated here.

\section{EXPERIMENTS}

Rubber press experiments on a double dome geometry (see figure 1) were performed at Stork/Fokker Special Products using Ten Cate Advanced Composites Cetex ${ }^{\circledR}$ material. The product shape consists of two intersecting hemispheres with different radii. 


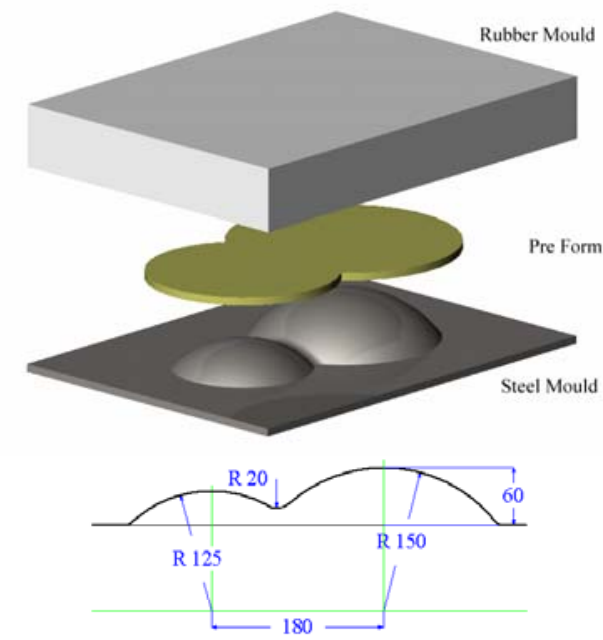

Fig 1: Rubber mould, pre form and steel mould for the double dome geometry (including cross-section)

Three configurations of Satin $8 \mathrm{H}$ glass fibre reinforced PolyPhenylene Sulfide (PPS) laminates were pressed, a $0^{\circ} / 90^{\circ}, 45^{\circ} /-45^{\circ}$ and a quasiisotropic (QI) $0^{\circ} / 90^{\circ} / 45^{\circ} / 45^{\circ}$ lay-up.

The laminates were heated up to $360{ }^{\circ} \mathrm{C}$ in the infrared oven during 220 seconds and immediately pressed. The constant press speed was set at 50 $\mathrm{mm} / \mathrm{sec}$. The experimental results are shown in figure 2 .
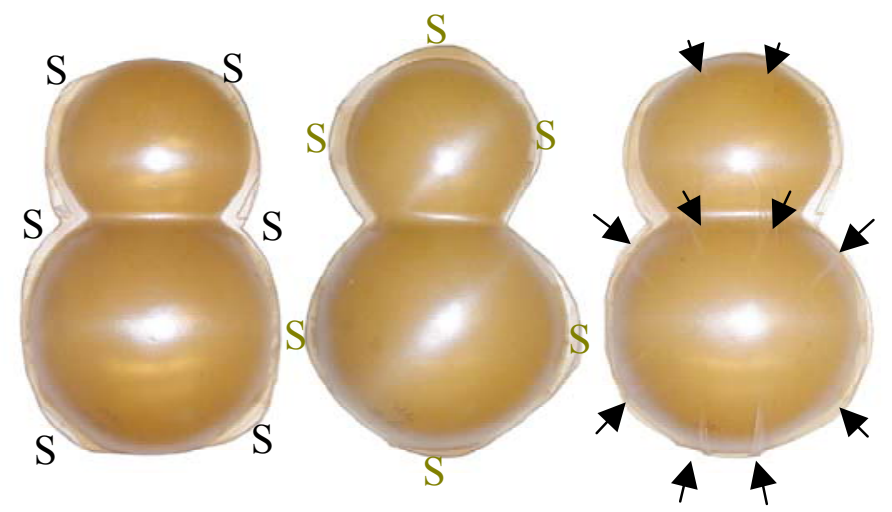

Fig 2: Rubber pressed 4-layered glass/PPS laminates, with $0^{\circ} / 90^{\circ}, 45^{\circ} /-45^{\circ}$ and a quasi-isotropic $0^{\circ} / 90^{\circ} / 45^{\circ} /-45^{\circ}$ lay-up

It is obvious from the experiments that the main fabric (shear) deformation occurs for both the $0^{\circ} / 90^{\circ}$ and the $45^{\circ} /-45^{\circ}$ lay-up in between the fibre directions (marked with an $\boldsymbol{S}$ in figure 2). However, the QI lay-up behaves completely different. This laminate wrinkles (black arrows in figure 2) and shows far less shear deformation than the other two laminates.

\section{SIMULATIONS}

\subsection{Geometrical Simulation}

For the geometrical drape simulation, the highest point strategy [2] was used. The initial point in the simulation was the top of the large hemisphere.

The initial fibre directions were equal to the fibre orientations in the experiments. With a mesh size of $3 \mathrm{~mm}$ the results for the simulations are shown in figure 3. Since geometrical draping methods drape one layer at the time only, the results for the laminates are obtained by sequential draping $0^{\circ} / 90^{\circ}$ and $/$ or $45^{\circ} /-45^{\circ}$ layers. The CPU time used for this simulation was 1 minute on a PC with an Athlon $850 \mathrm{MHz}$ processor.
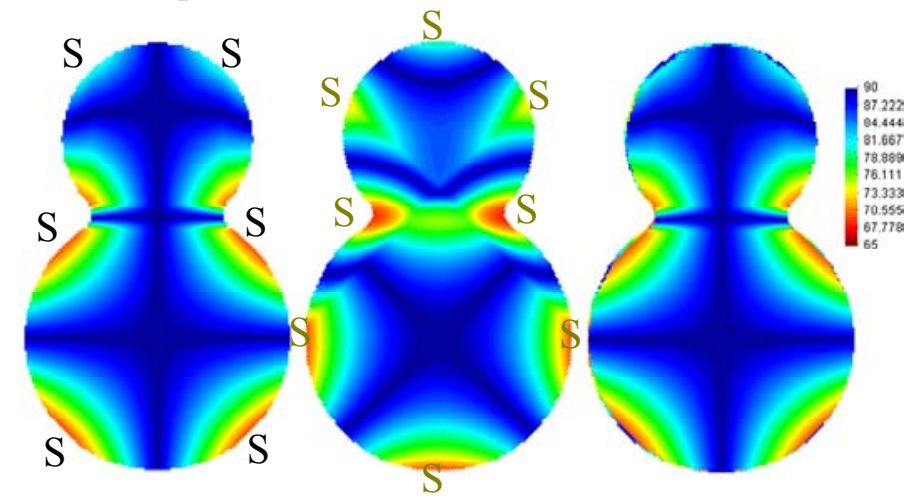

Fig 3: Enclosed fibre angle for geometrical drape simulation $\left(0^{\circ} / 90^{\circ}, 45^{\circ} / 45^{\circ}\right.$ and a QI lay-up)

\subsection{FE Simulation}

In the FE simulation, draping was simulated using an unstructured mesh of 4098 membrane elements. The average element size was approximately $4 \mathrm{~mm}$. The longitudinal modulus of the glass fibres (65 $\mathrm{GPa})$ was used as an input parameter for the Satin $8 \mathrm{H}$ glass fabric. The estimated input parameters for the PPS polymer matrix were: $\eta=100 \mathrm{~Pa} \cdot \mathrm{s}, \eta_{1}=30$ $\mathrm{Pa} \cdot \mathrm{s}, \eta_{2}=30 \mathrm{~Pa} \cdot \mathrm{s}, \eta_{3}=10 \mathrm{~Pa} \cdot \mathrm{s}$ and $\eta_{4}=10 \mathrm{~Pa} \cdot \mathrm{s}$. The fibre volume fraction was $50 \%$. The initial fibre directions were the same as in the experiments and the friction coefficient for contact was set at 33 $\mathrm{m} /(\mathrm{Pa} \cdot \mathrm{s})$. The press speed (affecting the viscous response) was set at $50 \mathrm{~mm} / \mathrm{s}$. As with the geometrical drape simulations, the results for the FE draping are obtained by sequential draping $0^{\circ} / 90^{\circ}$ and $45^{\circ} /-45^{\circ}$ layers. The results for the $\mathrm{FE}$ simulation are shown in figure 4.
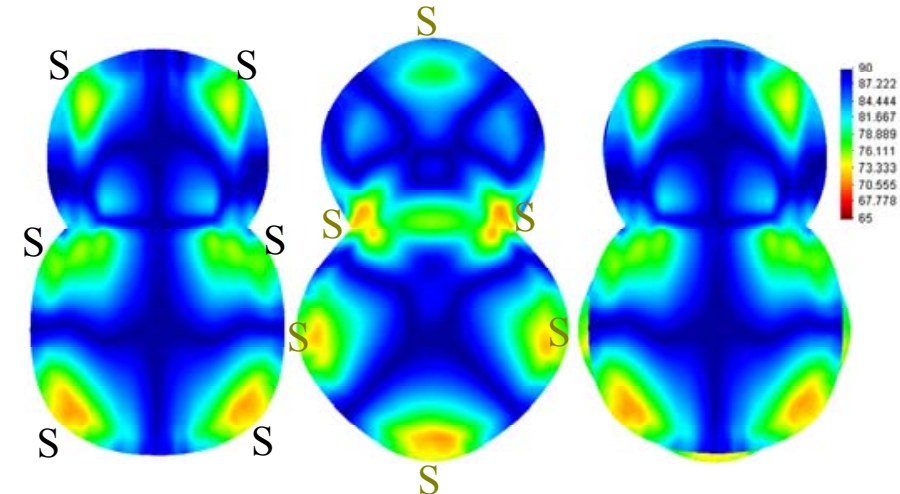

Fig 4: Enclosed fibre angle for FE drape simulation (perspective and top view)

The CPU time used for the FE simulation was 6 hours on a $\mathrm{PC}$ with an Athlon $850 \mathrm{MHz}$ processor. 


\section{DISCUSSION}

From the experiments it is clear that draping in the RPF process depends heavily on the lay-up of the fabric material. Draping $0^{\circ} / 90^{\circ}$ and $45^{\circ} /-45^{\circ}$ laminates resulted in smooth products. Wrinkles occurred in QI laminates during draping, as the different deformabilities of the separate layers is restricted by the interlaminar transverse shear stiffness of the laminate.

At first glance, the results of the geometrical and the FE drape simulation look similar to the experimental drape results for the $0^{\circ} / 90^{\circ}$ and $45^{\circ} /-45^{\circ}$ products. Both models predict large shear deformation of the fabric material at regions (denoted with an in $S$ in figure 3 and figure 4 ) between the fibre directions. The results at the smaller hemisphere however are quite different. For the QI lay-up both the geometrical and the FE drape simulations by adding the separate layer contributions did not predict these wrinkles. Iterative draping simulations seem no solution for draping multilayered preforms.

To evaluate the prediction of both the drape methods qualitatively, the enclosed fibre angle was measured in the area between the two hemispheres (the lower ridge). The results are depicted in figures 5 and 6 .

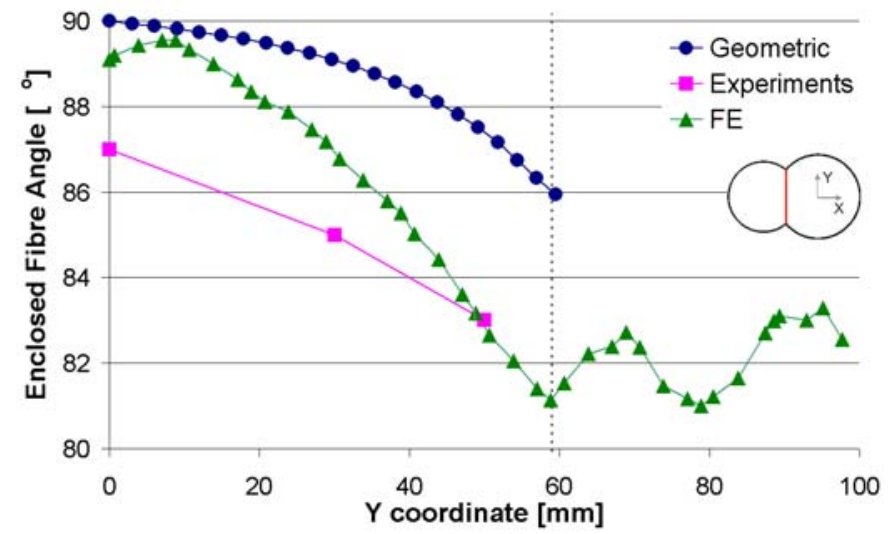

Fig 5: Enclosed fibre angle at ridge $\left(0^{\circ} / 90^{\circ}\right.$ lay-up $)$

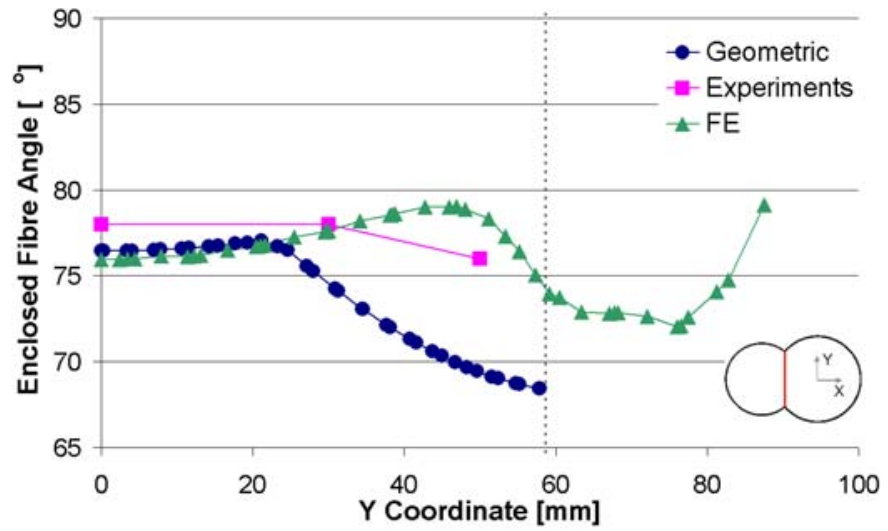

Fig 6: Enclosed fibre angle at ridge $\left(45^{\circ} / 45^{\circ}\right.$ lay-up)

In figures 5 and 6 , the dotted line represents the edge of the product. Both drape methods predict the correct trend, with a closer approximation of the FE simulations.

\section{CONCLUSION}

A material model was developed and implemented in the FE package DIEKA. It incorporates a biaxial fabric in a Newtonian viscous like matrix material. Drape simulations of the Rubber Press Forming process were performed with DIEKA and a geometrical draping method. The results were compared with experiments.

The experiments show a clear dependency between the drape behaviour and the laminate lay-up. Quasi Isotropic lay-ups drape significantly worse than $0^{\circ} / 90^{\circ}$ and $45^{\circ} /-45^{\circ}$ lay-ups. The QI lay-ups lead to wrinkling while the other lay-ups do not.

The FE simulations give the most accurate predictions. However, both methods do not predict wrinkling for the QI laminates. This is due to the sequential drape prediction.

Of course, if an analysis should be performed to gain a rough indication for the fabric deformation that might occur, the geometrical method is useful for its short CPU time.

\section{FUTURE WORK}

In the RPF-process a laminate of multiple fabric layers is pressed. Therefore, during pressing interlaminar shear deformation occurs. A material model that incorporates interlaminar shear behaviour is currently under development to model the draping process of multilayered composite materials more accurately.

\section{References}

[1] T. Meinders, B.D. Carleer, H. Vegter and J. Huétink, "Recent Developments In Finite Element Simulations Of The Deep Drawing Process", Shemet '97, Proceedings of the international conference at the University of Ulster, April 1997 [2] O.K. Bergsma, Three-dimensional Simulation of Fabric Draping (Ph.D. Thesis), Structures and Materials Laboratory, Delft University of Technology, 1995

[3] P. de Luca, P. Lefébure and A.K. Pickett, "Numerical and experimental investigation of some press forming parameters of two fibre reinforced thermoplastics: APC2-AS4 and PEIcetex", Composites: Part A, Volume 29A, pp.101-110, 1998

[4] S.P. McEntee and C.M. Ó Brádaigh, "Large deformation finite element modelling of single-curvature composite sheet forming with tool contact", Composites: Part A, Volume 29A, pp. $207-213,1998$

[5] A.J.M. Spencer, "Theory of fabric-reinforced viscous fluids”, Composites: Part A, Volume 31, p.1311-1321, 2000 